\title{
Aproximación a un sistema de medición de pedagogía ciudadana para niños y niñas escolares de Bogotá
}

Approximation to a measurement system of citizen pedagogy for scholar boys and girls of Bogotá

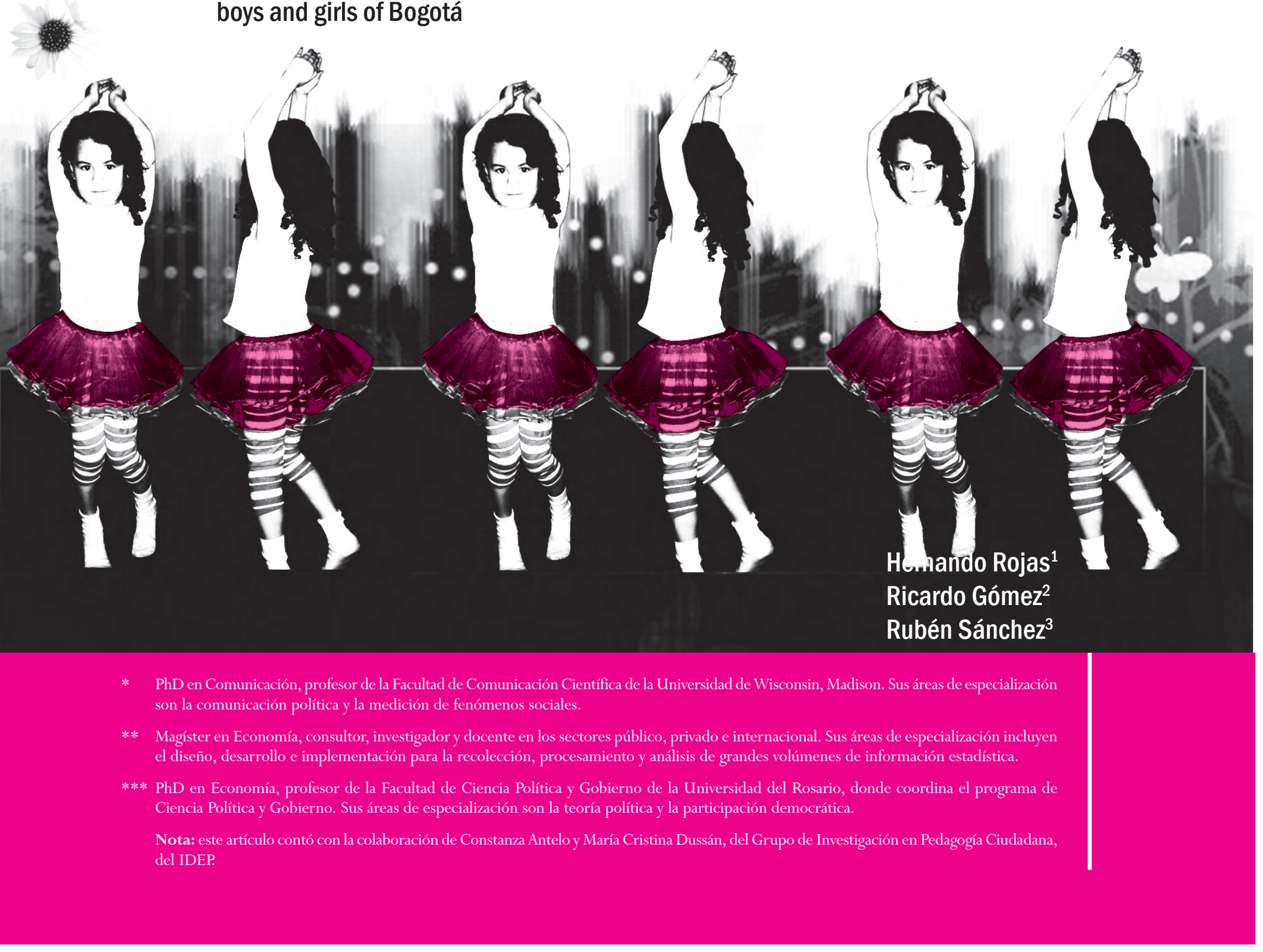


Resumen Reflexiones alrededor de un sistema de medición para realizar seguimiento y evaluación a un programa de pedagogía ciudadana a niños, niñas y adolescentes de Bogotá.

Palabras claves: patrimonio cultural, medio ambiente, espacio público, moralidad administrativa, ética pública, convivencia, pertenencia, patrimonio público, derechos, deberes, cultura ciudadana, pedagogía ciudadana.

Abstract Reflections around a measurement system to realize the following and the evaluation to a program of citizen pedagogy for boys, girls and adolescents of Bogota.

Keywords: cultural patrimony, environment, public space, administrative, morality, public ethics, coexistence, property, public patrimony, rights, duties, citizen culture, citizen pedagogy. 


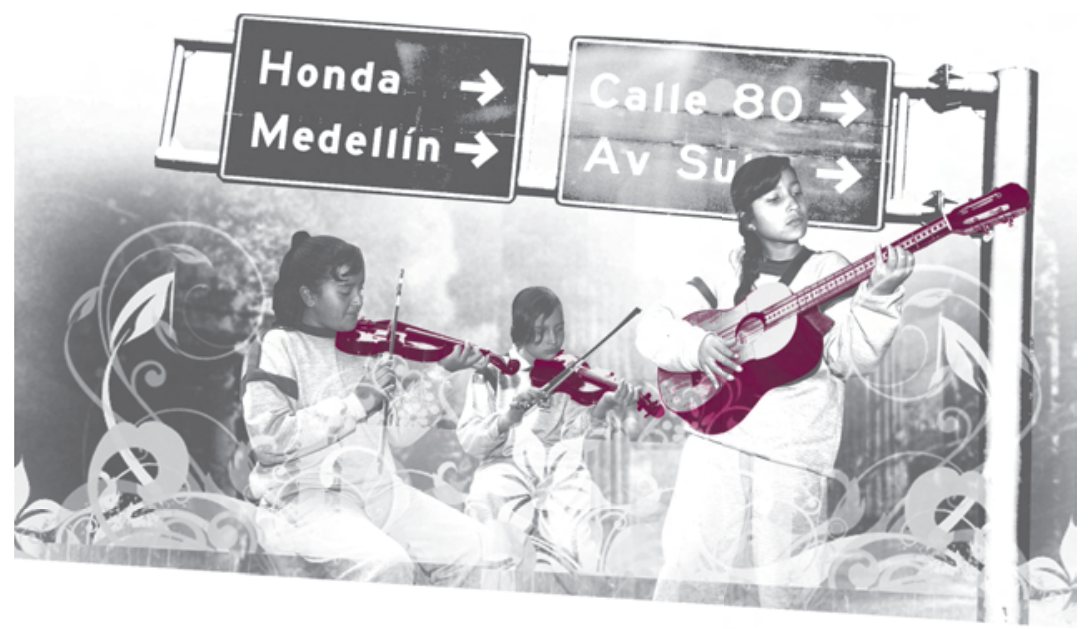

En los últimos años, ante la necesidad de fomentar los valores que fundamentan la democracia y la convivencia, diversas organizaciones públicas y privadas han promovido la idea de la educación para la ciudadanía y la investigación relacionada con la cultura ciudadana y la pedagogía de lo público. Prueba de ello son la promoción de la educación para la ciudadanía por parte de la Unesco, a través de su Decenio de las Naciones Unidas para la Educación en la esfera de los Derechos Humanos (1995-2004), y el proyecto sobre Educación para la Ciudadanía Democrática, impulsado por el Consejo de Europa, que culminó a finales de 2005. En Colombia, el Instituto para la Investigación Educativa y el Desarrollo Pedagógico, IDEP, ha propuesto, en el marco del Plan de Desarrollo Bogotá Positiva, adelantar un programa de Pedagogía Ciudadana cuyo propósito es promover la cultura ciudadana mediante la formación en ciudadanía de la población escolar del Distrito. El programa de Pedagogía Ciudadana busca desarrollar estrategias pedagógicas mediante las cuales se fortalezcan conocimientos, actitudes y comportamientos que mejoren la vida colectiva.

Como parte esencial de dicho programa de investigaciónacción se hace necesario establecer un sistema de medición que permita evaluar la situación general de la cultura ciudadana en niños y niñas de la ciudad, monitorear su evolución y evaluar programas específicos de intervención en la población objetivo. Este artículo resume las conclusiones de un grupo académico interdisciplinario constituido para la creación de un sistema de medición en cultura ciudadana para los niños y niñas de la ciudad. 


\section{Antecedentes}

Bogotá ha vivido, desde el Gobierno Distrital, un proceso de promoción de ciudadanía que, bajo diversos programas con énfasis complementarios y estrategias administrativas semejantes, ha sido reconocido distrital, nacional e internacionalmente por sus logros. De manera somera, este proceso puede caracterizarse de la siguiente manera:

\begin{tabular}{|c|c|c|}
\hline Gobierno & Programa & Énfasis \\
\hline Mockus 95-97 & Cultura Ciudadana & $\begin{array}{l}\text { Autorregulación social en espacios públicos y relación } \\
\text { funcionario-ciudadano. Énfasis en deberes. }\end{array}$ \\
\hline Peñalosa 98-00 & Saldo Pedagógico & Regulación gubernamental en espacios públicos. Énfasis en deberes. \\
\hline Mockus 01-03 & Cultura Ciudadana & $\begin{array}{l}\text { Autorregulación social en participación y cultura democrática. } \\
\text { Énfasis en deberes. }\end{array}$ \\
\hline Garzón 04-07 & Misión Bogotá & $\begin{array}{l}\text { Atención directa a necesidades individuales básicas. } \\
\text { Énfasis en derechos. }\end{array}$ \\
\hline Moreno 08-11 & Pedagogía Ciudadana & $\begin{array}{l}\text { Intervención en poblaciones escolarizadas. Énfasis en derechos y } \\
\text { deberes, particularmente en los llamados colectivos. }\end{array}$ \\
\hline
\end{tabular}

Estos procesos de promoción de ciudadanía han estado acompañados de variados ejercicios de observación, entre los que se destaca la medición de fenómenos sociales. El éxito de la medición de este tipo de fenómenos está vinculado a 1) claridad en la definición conceptual, 2) un proceso cuidadoso de operacionalización de dichos conceptos, 3) la solidez del diseño muestral y el trabajo de campo, 4) la regularidad o estabilidad de la medición, y 5) su relación con un marco de referencia socialmente aceptado.

A pesar de importantes avances en Bogotá relacionados con la medición de fenómenos sociales complejos, la inestabilidad en las definiciones conceptuales y en los marcos de referencia se ha traducido en inseguridad en los procesos de medición. ${ }^{1}$ Estas limitaciones del proceso pueden llegar a afectar la interpretación de la información recolectada, e incluso las acciones de intervención de la propia Administración Distrital. Por ello es importante detenerse en los aspectos conceptuales y operativos que sustentan el proyecto de Pedagogía Ciudadana.

1 Desde el Observatorio de Cultura Urbana de Bogotá se han realizado cinco encuestas para medir la cultura ciudadana de los bogotanos entre los años 2001 y 2007. Sin embargo, énfasis diferentes en cada encuesta reducen la posibilidad de llevar a cabo análisis longitudinales. 


\section{Cultura ciudadana}

Una de las dificultades es, precisamente, la definición de conceptos empleados en contextos diferentes.

En principio un ciudadano puede ser considerado como una persona que coexiste en una sociedad y la cultura como un factor de cohesión social que permite la interacción de los miembros de una sociedad. Dicha interacción se da en distintos planos de la realidad social, lo que permite hablar, en este orden de ideas, de cultura política y más específicamente de cultura ciudadana. En la medida en que la noción de cultura ciudadana lleva a cuestiones relativas a la sensibilización y el conocimiento de los derechos y deberes, es más amplio que el de cultura cívica, puesto que no se refiere únicamente al aprendizaje de hechos relacionados con las instituciones políticas sino que implica también la adquisición de competencias ligadas al quehacer de la ciudadanía.

Ahora bien, dado que como todo concepto político la noción de ciudadanía se enmarca en contextos cambiantes, es importante aclarar en el marco de qué modelo se maneja dicho concepto.

El modelo clásico de ciudadanía implica derechos cívicos, políticos y sociales que permiten la construcción de una sociedad "buena y libre" basada en tanta libertad e igualdad como sea posible (Marshall, 1950). En este modelo, cuyo caso ejemplar sería el francés, subyace una noción de identidad colectiva. Cuando ésta no está presente, aparece el modelo de Habsburgo, en el que se construye un sistema de derechos grupales diferenciados por grupos culturales y armonizados por un Gobierno ilustrado (Giesen y Eder, 2001).

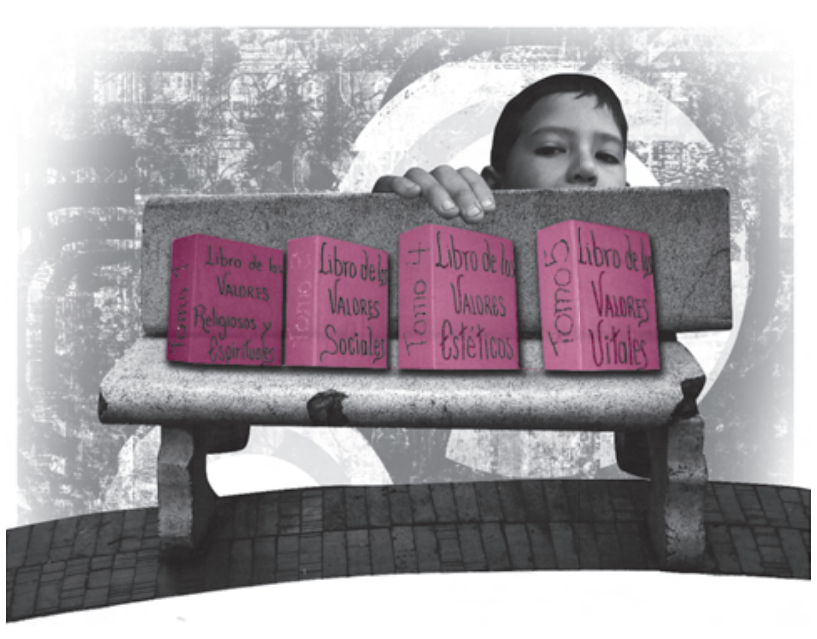

Históricamente el concepto de ciudadanía aparece como una demarcación de comunidades urbanas de iguales, en contraste con la tradicional distinción rural de amos y sirvientes. Este concepto de igualdad, inicialmente aplicado a grupos restringidos, se convierte con la Revolución francesa en una condición política y legal en permanente estado de expansión (por lo menos dentro de los límites del Estado-nación), que sirve de interfase entre el Gobierno y el pueblo (Giesen y Eder, 2001).

Esta relación entre Estado e individuo que se concreta en la ciudadanía ha sido descrita usando modelos diversos que, siguiendo a Giesen y Eder, corresponden a tres paradigmas: 


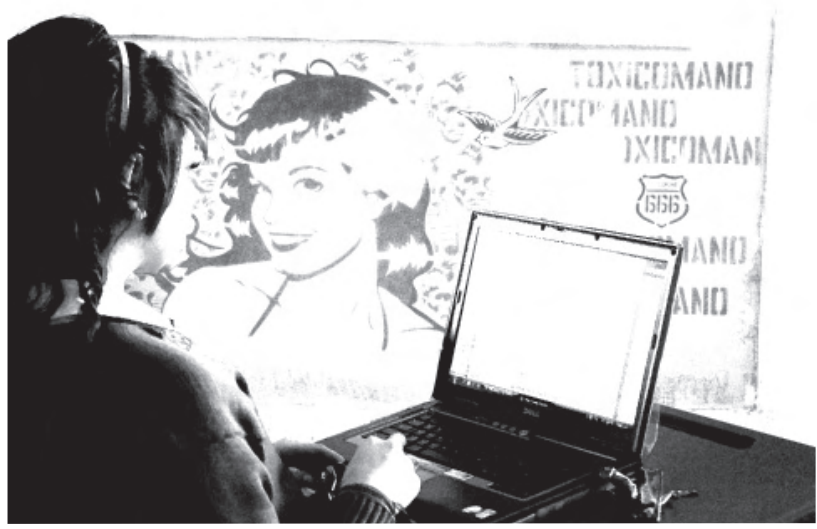

1. Paradigma individualista: que se concentra en las garantías legales para que los ciudadanos persigan sus intereses individuales (teoría liberal de la ciudadanía). En él se hace énfasis en los derechos del ciudadano, la igualdad jurídica y las garantías al acceso del aparato estatal. Dicho modelo busca integrar al ciudadano privilegiando el mercado a la acción estatal.

2. Paradigma comunitario: que enfatiza la participación política de todos en los debates de lo público. Este modelo de ciudadanía adiciona un fuerte componente de deberes ciudadanos y requiere un compromiso importante con causas comunes que sólo pueden ser realizadas con la participación permanente de los distintos actores sociales. Este modelo busca integrar al ciudadano en la esfera de lo público.

3. Paradigma de identidad colectiva: que enfatiza un legado de tradiciones o una cultura común. Más que en prácticas de inclusión hace énfasis en el sentido de pertenencia, afirmando una serie de valores comunes que promueven formas de participación emocional que privilegian la movilización simbólica acrítica. Este modelo asocia la noción de ciudadanía a emociones colectivas compartidas.

En el contexto de la Administración Distrital de Bogotá, el concepto de cultura ciudadana se introduce formalmente en el Plan de Desarrollo de Bogotá de 1995 (Formar Ciudad) como un "conjunto de costumbres, acciones y reglas mínimas compartidas que generan sentido de pertenencia, facilitan la convivencia urbana y conducen al respeto del patrimonio común y al reconocimiento de los deberes y derechos ciudadanos". ${ }^{2}$

De manera implícita, en la formulación de cultura ciudadana se encuentra el reconocimiento de tres sistemas de regulación del comportamiento humano, a saber: la ley, la moral y la cultura. Su contrastación con la realidad llevó a Antanas Mockus a diagnosticar una falta de armonía entre estos (Londoño, 2003), de forma tal que violar la ley podía ser cultural o moralmente aceptable. El tema de la cultura ciudadana se planteó como una estrategia para armonizar ley, moral y cultura desde la Administración Distrital, "desencadenando y coordinando acciones públicas y privadas que incidieran directamente en la manera como los ciudadanos perciben y usan los entornos sociales y urbanos y cómo se relacionan entre ellos en cada entorno". ${ }^{3}$

En su momento los promotores de la cultura ciudadana consideraron como una innovación importante asumir

2 Decreto 295 del 1 de junio de 1995. Formar Ciudad, Plan de desarrollo económico, social y de obras públicas para Santa Fe de Bogotá D. C., 1995-1998. Disponible en www.dapd.gov.co.

3 Decreto 295 del 1 de junio de 1995. Formar Ciudad, Plan de desarrollo económico, social y de obras públicas para Santa Fe de Bogotá D. C., 1995-1998. Disponible en www.dapd.gov.co. 
que un gobierno local podía intentar influir sobre la cultura y la conciencia y no solamente sobre la ley y sus mecanismos de aplicación (Bromberg, 2003; Mockus y Corzo, 2003).

Si bien estos programas de cultura ciudadana fueron novedosos en su aplicación, no se puede olvidar que los sistemas políticos han influido durante largo tiempo en la cultura y la conciencia. Lo han hecho, formalmente, a través de proyectos educativos de carácter público y políticas públicas, e informalmente, mediante praxis políticas determinadas.

A partir de 1998 algunos gobiernos distritales han enfocado sus esfuerzos de construcción de ciudadanía, en mayor o menor medida, guiados por el paradigma individualista, mientras que otros lo han hecho orientados por un paradigma comunitario. ${ }^{4}$ Esta dicotomía ha generado revisiones, discontinuidades y sinergias.

La respuesta a la pregunta de cuál es el tipo de ciudadano que se busca formar es, por supuesto, una respuesta de tipo político, y es natural que diferentes administraciones, elegidas con distintas agendas, enfaticen aspectos diversos. Sin embargo, y desde un punto de vista académico, las discontinuidades en el campo de la medición se vuelven problemáticas, pues no es posible analizar cambios que se quieren en una población a lo largo del tiempo. Este aspecto es particularmente complejo en el contexto de intervenciones sobre la cultura, donde, a diferencia del cambio reglado y muchas veces inmediato que la ley supone, puede tardar un periodo de tiempo mucho mayor.

Por ello, en este trabajo se adopta una tipología ciudadana que privilegia tanto el paradigma individualista (énfasis en derechos) como el comunitario (énfasis en deberes), y se entiende la pedagogía ciudadana como un proceso de socialización política que puede resultar en varios tipos de ciudadanos.

Una representación gráfica de esta tipología sobre los ejes de derechos y deberes sugiere que los ciudadanos podrían clasificarse de la siguiente manera:

\begin{tabular}{c|c|c|c|}
\multicolumn{2}{c|}{$\begin{array}{c}\text { Tipología del } \\
\text { ciudadano }\end{array}$} & \multicolumn{2}{c|}{ Énfasis en derechos } \\
\hline $\begin{array}{c}\text { Énfasis en } \\
\text { deberes }\end{array}$ & Bajo & Ciudadano anómico & Ciudadano legal \\
\hline & Ciudadano virtuoso & $\begin{array}{c}\text { Ciudadano } \\
\text { integral }\end{array}$ \\
\hline
\end{tabular}

Esta clasificación sirve para enmarcar los esfuerzos de socialización política en una sociedad determinada o de un gobierno en sus respectivos procesos de construcción ciudadana. Algunas ideologías políticas enfatizarán la socialización del ciudadano virtuoso, otras las del ciudadano legal y otras las del ciudadano integral, por lo que creemos que el sistema de medición del IDEP debe trascender los objetivos específicos de un gobierno. En su lugar, las mediciones futuras de cultura ciudadana deben asumirse como procesos de medición general que le sirvan a este y a futuros gobiernos de la ciudad, con 
base en una lógica de la comprobación empírica entre los tipos de ciudadano y su forma de vincularse a la vida en comunidad.

Por supuesto, ningún sistema de medición, por abarcante que sea, puede pretender medir todas las variables que afectan, o pueden llegar a afectar, todos los comportamientos sociales. Por ello se propone que el programa de medición en cultura ciudadana parta de un marco de referencia socialmente aceptado, en este caso el de la Constitución colombiana de 1991, producto del ejercicio más importante de democracia deliberativa que ha tenido la sociedad en los últimos años.

\section{Democracia deliberativa}

En la actualidad es común hablar de un giro deliberativo en la teoría democrática (Chambers, 2003; Delli-Carpini, Cook y Jacobs, 2004). Durante años prevaleció la noción de que en sociedades complejas la democracia giraba en torno a un problema de representación en la toma de decisiones colectivas. En un sistema de democracia representativa la toma de decisiones colectivas no son tomadas directamente por quienes forman parte de ella, sino por personas elegidas para este fin (Bobbio, 1994).

En una democracia representativa se privilegia un conjunto de reglas que determinan quién está autorizado para tomar las decisiones colectivas y bajo qué procedimientos. La condición de legitimidad de la democracia representativa es la existencia de dichas reglas y el respeto a las decisiones mayoritarias como vinculantes para toda la colectividad. En una democracia representativa la pregunta fundamental no es por el contenido de las decisiones, o si estas son moralmente aceptables, sino más bien por si se respetaron los procedimientos establecidos en las leyes.
La democracia representativa presupone la existencia de actores racionales que escogen entre alternativas para maximizar su utilidad (Joas \& Beckert, 2002). Sin embargo, es difícil mantener dicha presunción a la luz de las explicaciones pragmatistas de la construcción de la identidad individual y social.

John Dewey $(1882 ; 1888)$, con el concepto de habitus, sugiere que el sujeto político es constituido por una serie de interacciones con el medio ambiente y con los demás, por lo que tanto ese "individuo" como "sus intereses" son en realidad construcciones sociales. Desde esta perspectiva el actor social no está dado de antemano, sino que surge de las interacciones con los demás y por ello el sujeto es una negociación constante de la experiencia común (Mead, 1934; 1967).

Si aceptamos que los actores sociales y sus intereses son construcciones sociales, la lógica que subyace a la democracia representativa se desvanece, por cuanto los intereses individuales exigen un proceso de negociación constante y de construcción de significado común que sería indelegable. Este giro lingüístico en las ciencias sociales da lugar a una teoría deliberativa de la democracia que se fundamenta, ya no en un proceso reglado de toma de decisiones mayoritarias (aun cuando los procesos reglados mantienen su importancia), sino en un proceso de construcción de significado conjunto (Habermas, 1984; 1989).

En el caso colombiano, la Constitución Política de 1991 partió de un ejercicio deliberativo de un grupo de representantes y sentó las bases (a través de una serie de medidas participativas) para la transformación de una democracia representativa limitada en una democracia más robusta, con fundamentos deliberativos. 
Si bien la Constitución del 91 puede ser criticada (y su naturaleza deliberativa exige que estas críticas sean posibles y sean respondidas con racionalidad comunicativa), esta se convierte en el marco socialmente aceptado por excelencia que puede servir de referencia tanto a la administración para sus intervenciones, como a los procesos de medición de fenómenos sociales.

Si el individuo es una construcción social, el ciudadano también lo es por definición. Esto quiere decir que la ciudadanía se construye a través de interacciones formales e informales que pueden y deben ser intervenidas desde lo público. Por supuesto, muchas de estas interacciones van más allá del ámbito de la educación formal, pero tal como lo sugieren los estudios de socialización política, la escuela es un escenario privilegiado en la construcción de ciudadanía.

\section{La socialización política}

El estudio empírico de la socialización política comienza en los años cincuenta como parte de la revolución cognitiva en las ciencias sociales, que comienza a favorecer la interpretación del comportamiento político como un comportamiento aprendido (Niemi y Hepburn, 1995). No es extraño, entonces, que para entender el comportamiento político del adulto se comenzaran a explorar las actitudes y comportamientos de los niños y los adolescentes.

Muy pronto las investigaciones revelaron que los niños exhibían lo que entonces se conceptualizó como preactitudes, que incluían sentimientos de patriotismo (Connell, 1971), percepciones sobre otras naciones (Jahoda, 1963) y prejuicios raciales (Stevenson y Stuart, 1958). Incluso niños muy pequeños (de 6 a 9 años, en

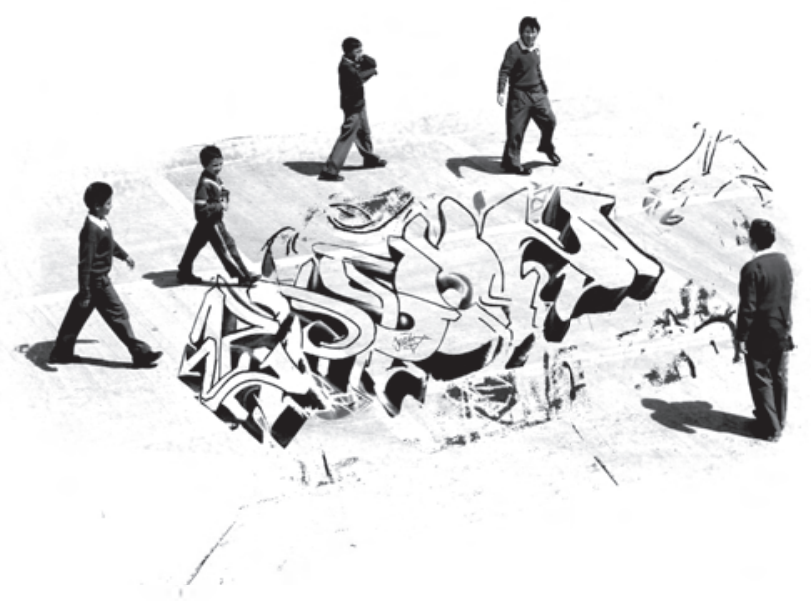

los estudios de Easton y Dennis, 1969) ya articulaban preferencias y sentimientos sobre el Gobierno y los partidos políticos.

Easton y Dennis (1969) definieron la socialización política como una serie de procesos de desarrollo mediante los cuales una persona adquiere unas orientaciones políticas y unos patrones de comportamiento político. Inicialmente se consideraba a la familia y a la escuela como los agentes de socialización por excelencia, pero la definición de Easton y Dennis permitió incluir nuevos agentes de socialización como medios de comunicación (Livingstone, 2002) y pares; así mismo, desligó la socialización de unas consecuencias sistémicas predeterminadas (preservar o cambiar el sistema) que deben ser más bien establecidas contextualmente. 
Cinco décadas de investigación en socialización política sugieren que:

1. A pesar del surgimiento de nuevos agentes de socialización, la familia conserva su importancia. Es así como las actitudes políticas de los padres determinan en gran medida las actitudes de sus hijos (Glass, Bengston y Dunham, 1986; Hirschfeld, 1995)

2. La escuela se convierte en lugar privilegiado de la socialización política (Easton y Dennis, 1969), pero, aunque se trate de una educación universal, los niños se socializan de forma diferenciada. De hecho, Easton y Dennis muestran cómo en el segundo año de primaria las opiniones de los niños sobre si el Congreso o el presidente son más importantes para gobernar un país, fueron iguales en distintos estratos socioculturales, pero que, con el paso del tiempo, los niños de estratos sociales más altos comienzan a valorar más al Congreso y los de estratos más bajos al presidente. Litt (1963), analizando el contenido de las clases de cívica entre estratos, muestra que los niños son objeto de diferentes procesos de socialización política, dependiendo del estrato socioeconómico al que pertenezcan, lo que los lleva a comprender de variada manera la participación política.

3. La socialización que ocurre en la casa y en la escuela se ve contextualizada por un momento histórico y político que produce cambios intergeneracionales (Jennings, 1987).

4. Los pares adquieren importancia como agentes de socialización, especialmente en algunos temas que cobran relevancia para el grupo de pares (Tedin, 1980).
5. Los medios de comunicación masiva se consolidan como agentes de socialización política. En un primer tiempo, la televisión privatizó parte de la vida cívica, desplazando a la comunidad y centrándola en la familia, pero ahora, cuando las nuevas tecnologías de comunicación refuerzan la individualización del consumo de medios, es el niño quien comienza a ser "privatizado" en el seno de su propia familia. El influjo de los medios es complejo y a veces contradictorio, pues si bien su contenido puede 
generar escepticismo sobre ciertas instituciones democráticas, también promueven la idea del niño como agente más que como sujeto de obediencia y disciplina, lo que propendería a la democratización de la familia (Livingstone, 2002).

6. La conceptualización inicial de la socialización política como un proceso de arriba hacia abajo (Butler y Stokes, 1974) ha sido remplazada por modelos más complejos que incluyen una conceptualización más activa del niño, e inclusive procesos de socialización de abajo hacia arriba, en los que son los niños quienes socializan a sus padres (actitudes sobre temas ecológicos son un buen ejemplo de lo que algunos han llamado la socialización revertida. Ver McDevitt y Chaffee, 2002).

En efecto, la ciudadanía, como cualquier rol, requiere para que se construya su ejercicio, un proceso de socialización y de formación que supera el conocimiento de los derechos de los que se es titular y supone la existencia de espacios que otorguen la oportunidad de constituirse en sujetos de derechos y responsabilidades en la práctica cotidiana.

Si bien en el pasado las administraciones distritales privilegiaron en sus intervenciones de cultura ciudadana a poblaciones adultas, se ha evidenciado que para la niñez los espacios educativos son escenarios privilegiados para la construcción de ciudadanía, así como para una evaluación rigurosa de mejores prácticas. Esta circunstancia ha llevado al IDEP a evaluar el estado de cultura ciudadana en niños y niñas de los colegios públicos y privados de Bogotá, lo cual plantea de entrada, como problema, el tema de la medición.

\section{Propuesta de medición}

La estrategia de medición propuesta para pedagogía ciudadana combina un diseño transversal con muestra probabilística para la población general, con diseños cuasi experimentales para poblaciones intervenidas y la aplicación de un instrumento único de medición para ambos diseños.

\section{Instrumento de medición}

Específicamente, se elaboró un instrumento para el final del segundo ciclo educativo (cuarto grado) que permite medir disposiciones en cultura ciudadana en los ámbitos cognitivo (conocimiento), actitudinal (valores y afectos) y de comportamiento (prácticas). Igualmente, se optó por la aplicación de un formulario único, en lugar de desarrollar uno de medición para cada ciclo o cada actividad de intervención. Esta recomendación se hizo ante la dificultad analítica para establecer equivalencias cuando se dispone de más de un formulario.

Teniendo en cuenta que en su fase inicial las intervenciones del IDEP se concentrarán en el segundo ciclo, se desarrolló un instrumento de medición para el mismo y se sugirió a la administración que, a medida que incluya nuevos ciclos, elabore un cuestionario para el final de cada uno de ellos. Dichos cuestionarios deberán ajustarse en su estructura al desarrollado para el ciclo dos, buscando, sin embargo, que las mediciones sean sensibles a las mayores capacidades cognitivas, actitudinales y de comportamiento de los niños y niñas de cada ciclo.

Debe tenerse en cuenta que tales instrumentos servirán especialmente para comparar niños y niñas equivalentes, es decir, del mismo ciclo, más que para monitorear 
la evolución entre ciclos (por la dificultad en la homologación de cuestionarios). Es posible que en el futuro, después de múltiples aplicaciones y utilizando técnicas de panel, el IDEP logre dicha homologación de instrumentos. El cuestionario desarrollado está diseñado para la autoaplicación, pero en lo posible, especialmente para la medición general (líneas de base y tendencias); se recomienda que sea diligenciado por un entrevistador entrenado en su manejo.

\section{Indicadores}

En vista de que el resultado de la formación en cultura ciudadana no depende solamente de las decisiones políticas de los gobernantes sino también de las decisiones cotidianas que corresponden al ciudadano como consumidor, habitante de barrio, usuario de servicios, miembro de asociaciones o responsable de un quehacer profesional, es importante conocer en qué nivel se encuentran los individuos con respecto al desarrollo de su cultura ciudadana antes de concebir estrategias tendientes a su fortalecimiento.

Dado que la aplicación de métodos cuantitativos no provee directamente elementos que permitan evaluar los niveles de cultura ciudadana de un individuo o grupo social al resultar muy subjetivo el establecimiento de criterios como alto, bajo o muy bajo, la utilización de indicadores que permitan apreciar las variaciones en el tiempo es fundamental.

La expresión de los avances en un tema como el de la cultura ciudadana se aprecia fácilmente recurriendo a indicadores de la vida cotidiana. El correcto uso de los lavabos públicos, por ejemplo, o el trato a los pupitres en la escuela, pueden ser buenos indicadores de cultura y convivencia. Sin embargo, cada uno de los indicadores debe ser leído sin perder de vista sus limitaciones y el contexto correspondiente. En el caso de la mala utilización de los lavabos públicos como indicador inmediato, pueden perderse de vista otros problemas sociales como la pobreza y la inequidad en el sentido de que la utilización de aquellos está asociada directamente con la calidad de la vivienda, el hacinamiento, la no disponibilidad de servicios adecuados y la falta de educación en condiciones de pobreza.

Para la medición del nivel de la cultura ciudadana a través de la evaluación de sus componentes, el contar con indicadores es un aspecto fundamental ya que estos constituyen parámetros obtenidos mediante la combinación de información sobre una situación dada, que brindan señales asociadas a la dinámica, avance o retroceso del proceso en formación de cultura ciudadana. 


\section{Línea de base y tendencias}

La línea de base para el proyecto de investigación-acción se elaborará a partir de la aplicación del instrumento de medición de una muestra representativa de la población escolar de cuarto grado. Dicha línea de base establecerá las disposiciones ciudadanas en el año 2009 (antes del comienzo de las intervenciones) de los niños y niñas de la ciudad que cursan dicho grado, constituyéndose en un estudio transversal de esa población. Esta medición debe repetirse cada dos años con una muestra de niños y niñas seleccionada utilizando la misma metodología que para la línea de base, de forma que, a partir de múltiples estudios transversales (2009, 2011, 2013, 2015), se pueda construir una tendencia longitudinal que verifique los avances o retrocesos de la ciudad en materia de disposiciones ciudadanas en un ciclo educativo determinado.

Esta lógica de medición debería mantenerse al abordar nuevos ciclos pedagógicos, como se visualiza en la gráfica siguiente:

\begin{tabular}{c|c|c|c|c|c}
\hline Ciclo & Medición & $\begin{array}{c}\text { Línea de } \\
\text { base }\end{array}$ & Serie & Serie & Serie \\
\hline Dos (3 y 4) & Grado 4 & $\mathbf{2 0 0 9}$ & 2011 & 2013 & 2015 \\
\hline Tres (5 a 7) & Grado 7 & & $\mathbf{2 0 1 1}$ & 2013 & 2015 \\
\hline $\begin{array}{c}\text { Cuarto (8 a 9) } \\
\text { Quinto (10 } \\
\text { y 11) }\end{array}$ & Grado 9 & & & $\mathbf{2 0 1 3}$ & 2015 \\
\hline
\end{tabular}

La estrategia propuesta permitirá monitorear las tendencias en cada ciclo, en cuanto a la evolución de las disposiciones ciudadanas, y debería reflejar el éxito de las intervenciones propuestas por el IDEP (en la medida en que estas hayan sido acogidas y masificadas por la SED), así como la ocurrencia de factores externos que modifiquen positiva o negativamente las disposiciones ciudadanas en el Distrito.
El éxito de la política general de medición está dado por la representatividad de las muestras y la consistencia en la aplicación de los instrumentos de medición en el tiempo. La muestra probabilística ideal es multietápica por conglomerados, tomando al colegio como unidad básica de muestreo. Los integrantes finales de la muestra, escogidos a partir de listas disponibles en cada colegio, serán seleccionados aleatoriamente entre colegios también previamente escogidos al azar (teniendo en cuenta el tamaño de los mismos para mantener uniforme la probabilidad de ser seleccionados en la muestra y ésta deberá ser estratificada, con el fin de garantizar representatividad por régimen público/privado y calidad). Es importante que la selección en el colegio también sea aleatoria, para lo cual el entrevistador deberá estar dotado con una tabla de números aleatorios y un protocolo de selección. En ningún caso el colegio seleccionará al alumno a ser encuestado.

\section{Diseño cuasi experimental}

En forma adicional a las mediciones generales, el instrumento de medición desarrollado puede ser utilizado por el IDEP para evaluar las intervenciones específicas realizadas para mejorar las disposiciones ciudadanas de los niños y niñas, pudiendo así recomendar a la Secretaría de Educación Distrital las prácticas que deban ser masificadas. Para ello se considera apropiado un diseño cuasi experimental de grupos no equivalentes con el grupo de control, que debe acompañar toda estrategia de intervención, si bien desde el punto de vista investigativo un diseño verdaderamente experimental (es decir, con asignación aleatoria a los grupos) como el de postintervención con grupo de control sería deseable, de manera que: 


$\begin{array}{lll}\text { Grupo } 1 & \mathrm{X} & \mathrm{O} 1 \\ \text { Grupo } 2 & \mathrm{X} & \mathrm{O} 1^{5}\end{array}$

La logística de dicha aplicación la hace improbable para colegios organizados por cursos. Por ello se considera idóneo el diseño cuasi experimental para grupos que ocurren naturalmente (cursos), que son expuestos o no a la intervención (la decisión de qué curso es expuesto a la intervención y cuál permanece como control debe ser aleatoria) y observados antes y después de la intervención propuesta. De modo que:

$\begin{array}{llll}\text { Curso } 1 & \text { O1 } & X & \text { O2 } \\ \text { Curso } 2 & \text { O1 } & & \text { O2 }\end{array}$

El diseño propuesto es robusto, pues permite descartar problemas de validez por historia, maduración, prueba, instrumentación, selección y mortalidad, mas no así de instrumentación ${ }^{6}$.

Se prevé entonces que antes de la intervención el instrumento de medición sea aplicado a la población intervenida y a una población equivalente que no sea objeto de intervención, y una vez se haya terminado este último proceso, el instrumento sea aplicado una vez más a los dos grupos. Como a pesar de los esfuerzos que se hagan por establecer a priori la equivalencia de los dos grupos estos pueden no ser equivalentes, se impone la observación previa del grupo de control (01), de forma tal que esta equivalencia pueda establecerse empíricamente. Desigualdades posteriores (02) podrán ser entonces atribuidas a la intervención.
En suma, la noción de cultura ciudadana lleva a plantear cuestiones relativas a la sensibilización y al conocimiento de los derechos y deberes, así como a valores cívicos como la democracia, los derechos humanos, la igualdad, la solidaridad, la tolerancia, el patrimonio público, el medio ambiente y la moralidad administrativa. Ahora bien, el fortalecimiento de dichos aspectos y, por ende, de la cultura ciudadana, requiere la construcción de un sistema de medición que permita evaluar la situación en un momento dado y para ello contar con los instrumentos adecuados.

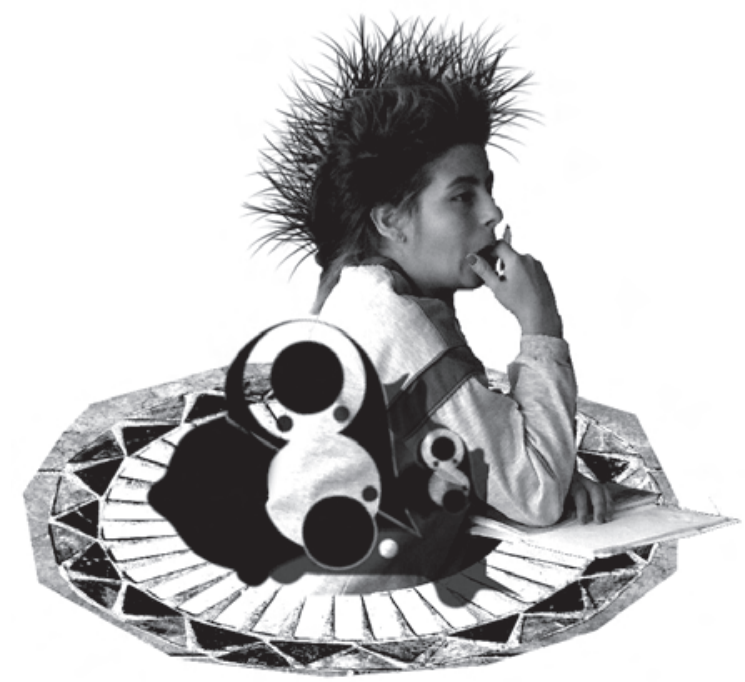

5 Donde $\mathrm{X}$ equivale a la intervención y $\mathrm{O}$ a la observación.

6 Para una explicación más detallada, ver Campbell D. T.; Stanley, J. C., Experimental and Quasi-Experimental Designs for Research. Houghton Mifflin Company, Boston, 1963. 

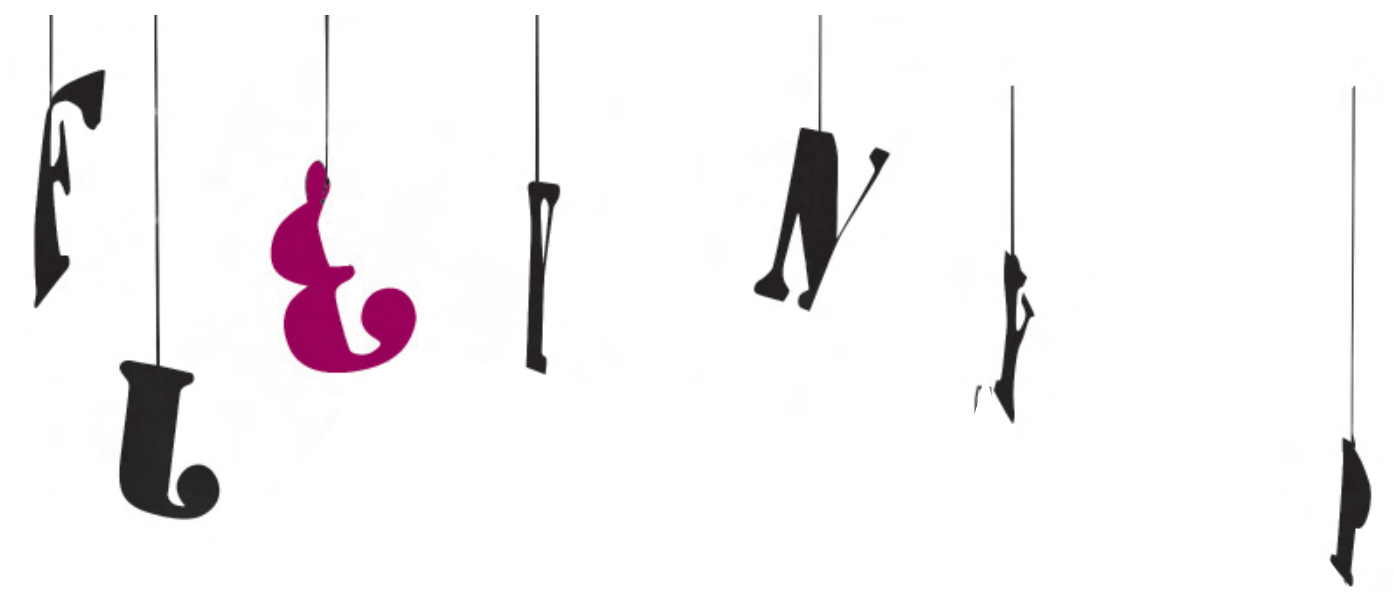

\section{Bibliografía}

Bobbio, N. El futuro de la democracia, Bogotá, Fondo de Cultura Económica, 1994.

Bromberg, P. De ingenieros y profetas: transformaciones dirigidas de comportamientos colectivos, en Educación Hoy, 2003, 32: 25-70.

Butler, D.; Stokes, D. Political change in Britain, London, McMillan, 1974.

Chambers, S. "Deliberative democratic theory", en Annual Review of Political Science, 2003, 6: 307-326.

Connell, R. W. The child's construction of politics, Melbourne, Melbourne University Press, 1971.

Delli-Carpini, M. X.; Cook, F. L.; Jacobs, L. R. "Public deliberation, discursive participation and citizen engagement: A Review of the Empirical Literature", en Annual Review of Political Science, 2004, 7: 315-344.

Dewey, J. Human Nature and Conduct, Carbondale, Southern Illinois University Press, 1922; 1988.

Easton, D.; Dennis, J. Children in the political system, New York, McGraw-Hill, 1969.

Giesen, B.; Eder, K. “European citizenship: An avenue for the social integration of Europe”, en K. Eder y B. Giesen (editores), European Citizenship between National Legacies and Postnational Projects, Oxford: Oxford University Press, 2001.

Glass, J.; Bengston, V. L.; Dunham, C. C. "Attitude similarity in three-generation families: socialization, status inheritance or reciprocal influence?”, en American Sociological Review, 1986, 51: 685-699.

Habermas, J. The Theory of Communicative Action: Reason and the Rationalization of Society, Boston, Beacon Press, 1984. 1989. .The Theory of Communicative Action. Lifeworld and System A critique of Functionalist Reason, Boston, Beacon Press,

Hirschfeld, L. A. "The inheritability of identity: Children's understanding of the cultural biology of race”, en Child Development, 1995, 66: 1418-1437.

Jahoda, G. "The development of children's ideas about country and nationality", en British Journal of Educational Psychology, 1963, 33: 143-53.

Joas, H.; Beckert, J. "A theory of action: Pragmatism and the creativity of action”, en Transactional Viewpoints, 2002, 1: 410-413. 
Jennings, K. "Residues of a movement: the aging of the American protest generation", en American Political Science Review, 1987, 81: 367-382.

Livingstone, S. Young People and New Media: Childhood and the Changing Media Environment, London, Sage, 2002.

Londoño, R. "Líneas de investigación e intervención en los programas de cultura ciudadana de Bogotá (1995-1997, 2001-2004)", en Pensar Iberoamérica, 2003, 4. (Disponible en http://www.oei.es/pensariberoamerica/ric04a08.htm).

Manheim, K. “The problem of generations”, en P. G. Altbach y R. S. Laufer (editores), The New Pilgrims, New York, David McKay, 1972, pp. 101-138.

Marshall, T. H. Citizenship and social class and other essays, Cambridge, Cambridge University Press, 1950.

McDevitt, M.; Chaffee, S. H. "From top-down to trickle-up influence: Revisiting assumptions about the family in political socialization”, en Political Communication, 2002, 19: 281-301.

Mead, G. H. Mind, Self \& Society: From the Standpoint of a Social Behaviorist, Chicago, University of Chicago Press, $1934 / 1967$.

Mockus, A.; Corzo, J. Cumplir para convivir, factores de convivencia y su relación con normas y acuerdos, Instituto de Estudios Políticos y Relaciones Internacionales, Universidad Nacional de Colombia, 2003.

Niemi, R.; Hepburn, M. “The rebirth of political socialization”, en Perspectives on Political Science, 1995, 24(1), 24(1): 7. (Retrieved December 14, 2008, from Academic Search Elite database).

Stevenson, H.; STUART, E. "A developmental study of racial awareness in young children", en Child Development 1958, 29: 399-409.

Tedin, K. L. "Assessing peer and parental influence on political attitudes", en American Journal of Political Science, 1980, 24: 136-154. 